\title{
Intermodal parametric gain of degenerate four wave mixing in large mode area hybrid
} photonic crystal fibers

\author{
Petersen, Sidsel Rübner; Lægsgaard, Jesper; Alkeskjold, Thomas Tanggaard
}

Published in:

Nonlinear Optics Technical Digest

Publication date:

2013

Document Version

Publisher's PDF, also known as Version of record

Link back to DTU Orbit

Citation (APA):

Petersen, S. R., Lægsgaard, J., \& Alkeskjold, T. T. (2013). Intermodal parametric gain of degenerate four wave mixing in large mode area hybrid photonic crystal fibers. In Nonlinear Optics Technical Digest (pp. NW4A.08). Optical Society of America.

\section{General rights}

Copyright and moral rights for the publications made accessible in the public portal are retained by the authors and/or other copyright owners and it is a condition of accessing publications that users recognise and abide by the legal requirements associated with these rights.

- Users may download and print one copy of any publication from the public portal for the purpose of private study or research.

- You may not further distribute the material or use it for any profit-making activity or commercial gain

- You may freely distribute the URL identifying the publication in the public portal 


\title{
Intermodal parametric gain of degenerate four wave mixing in large mode area hybrid photonic crystal fibers
}

\author{
Sidsel R. Petersen ${ }^{1, *}$, Jesper Lagsgaard ${ }^{1}$, Thomas T. Alkeskjold ${ }^{2}$ \\ ${ }^{1}$ DTU Fotonik, Technical University of Denmark, Ørsteds Plads Building 343, 2800 Kgs. Lyngby, Denmark \\ ${ }^{2}$ NKT Photonics A/S, Blokken 84, 3460 Birkerød, Denmark \\ *srpe@fotonik.dtu.dk
}

\begin{abstract}
Intermodal degenerate four wave mixing (FWM) is investigated numerically in large mode area hybrid photonic crystal fibers. The dispersion is controlled independently of core size, and thus allows for power scaling of the FWM process.
\end{abstract}

(C) 2013 Optical Society of America

OCIS codes: (190.4380) Nonlinear optics, four-wave mixing; (060.5295) Photonic crystal fibers.

\section{Introduction}

Control of the group velocity dispersion (GVD) is the key to control the spectral position of the maximum gain of a FWM process in silica fibers. Tailoring of the GVD is possible in photonic crystal fibers (PCFs), since the zero GVD wavelength (ZDW) scales with core size [1]. However, for power scaling a large mode area (LMA) is needed, which is incompatible with the GVD control in standard PCFs through core scaling, since the material dispersion dominates in large core fibers. In this work a hybrid LMA PCF is considered, with a cladding consisting of airholes and high-index inclusions. A microscope image of such a fiber is seen in Fig. 1a. The high-index inclusions give rise to a photonic bandgap (PBG) effect, and adjustment of the airhole diameters allow for single mode (SM) guidance in a LMA core. The diameter size of the high-index inclusions on one side of the core is reduced with respect to the diameter size on the other side of the core, resulting in an asymmetric design, providing control of blue- and red spectral edge of the PBGs [2]. In Fig. 1b the calculated overlap integral of the fundamental mode (FM) polarized along the Ge-rods with the core region is shown. The overlap integral has been calculated in steps of $0.1 \mathrm{~nm}$ with a full-vector modal solver based on the finite element method. In Fig. 1c the calculated group velocity dispersion parameter (D) of the transmission band $990 \mathrm{~nm}-1070 \mathrm{~nm}$ is shown. The Zero Dispersion Wavelength (ZDW) is positioned at $1054.5 \mathrm{~nm}$, shorter wavelengths lie in the normal dispersion regime, while the dispersion is anomalous for longer wavelengths. Near the bandedges the curve diverges substantially. Large values of normal and anomalous GVD is thus experienced on the edges of the PBGs, providing control of the GVD independently of core size.

\section{Degenerate four wave mixing}

Degenerate four wave mixing is a third order nonlinear process. In the process two pump photons are annihilated and one so-called signal and idler photon are created. For the process to take place, two conditions must be satisfied. The first is energy conservation, which is given by $2 \omega_{p}=\omega_{s}+\omega_{i}$, where $\omega_{p}$ is the pump photon, $\omega_{s}$ the signal photon, and $\omega_{i}$ the idler photon. The second condition is phase matching, which has a linear and nonlinear contribution. The linear contribution is given by $\Delta \beta=\beta\left(\omega_{s}\right)+\beta\left(\omega_{i}\right)-2 \beta\left(\omega_{p}\right)$, where $\beta\left(\omega_{s}\right), \beta\left(\omega_{i}\right)$, and $\beta\left(\omega_{p}\right)$ is the propagation constant at signal, idler, and pump frequency, respectively, and the nonlinear contribution arises from the Kerr effect.

\subsection{Parametric gain}

The parametric gain can be calculated from a set of coupled differential equations $[3,4]$. Overlap integrals of the interacting pump-, signal-, and idler- fields are included in these equations. These overlap integrals are typically assumed to be equal, which is a good approximation in e.g. endlessly single mode PCFs, where the spatial mode distribution is approximately the same at all wavelengths. However, when PBG guidance is present, the spatial mode distribution differs substantially, and the overlap integrals cannot be approximated by any simple expression.

If a pump power much higher than the signal and idler power is assumed, and the pump remains undepleted in the 


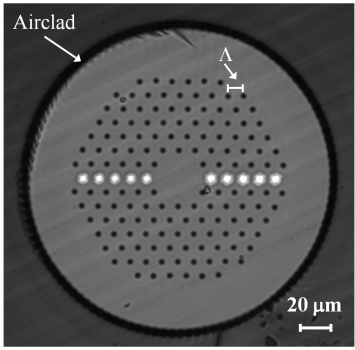

(a)

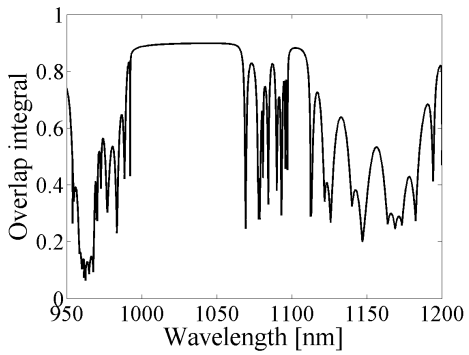

(b)

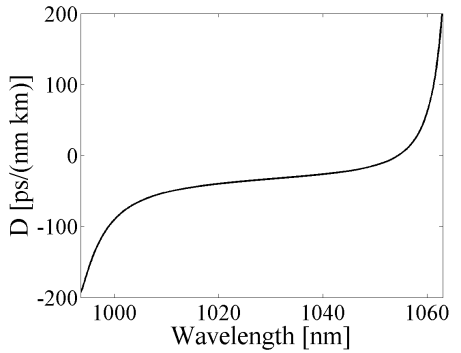

(c)

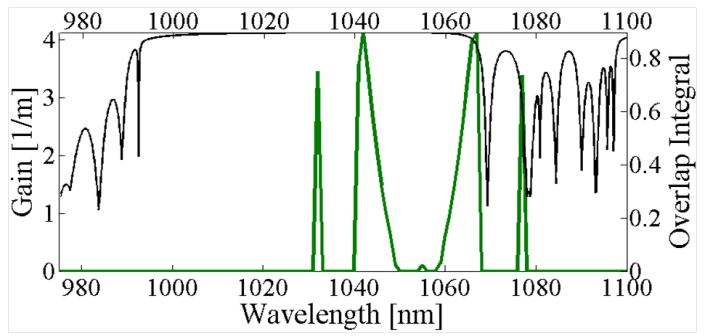

(d)

Fig. 1: (a) Microscope image of the double-clad large mode area hybrid photonic crystal fiber. The inner cladding consists of airholes (black circles) with hole-to-hole spacing $\Lambda$ and seven missing airholes define the core region. One row of airholes is replaced with high-index Germanium-doped silica rods of two sizes (white circles). (b) Calculated overlap integral of the fundamental mode polarised along the Germanium-doped silica rods with the core region. (c) Calculated group velocity dispersion in the transmission band $995 \mathrm{~nm}-1065 \mathrm{~nm}$. (d) Calculated parametric gain (green curve, y-axis on the left) for the case where the signal and idler are generated in the fundamental mode. The overlap integral is also shown (black curve, $\mathrm{y}$-axis on the right).

FWM process, an approximate expression for the parametric gain can be obtained. Following a similar approach as in Sec. 10.2.2 in [4] the effective phase mismatch, $\kappa$, and approximate parametric gain, $g$, can be found:

$$
\begin{aligned}
\kappa & =\Delta \beta+2 \frac{n_{2} \omega_{p}}{c} P_{p}\left(f_{s p}+f_{i p}-f_{p p}\right), \\
g & =\sqrt{\left(\frac{n_{2} \omega_{p}}{c} P_{p} f_{\text {sipp }}\right)^{2}-\left(\frac{\kappa}{2}\right)^{2}},
\end{aligned}
$$

where $n_{2}$ is the nonlinear-index coefficient, $c$ is the speed of light, $P_{p}$ is the pump power, and $f_{j k},(j k=s p, i p, p p)$, and $f_{\text {sipp }}$, are the field overlap integrals defined in [4] with the indices representing the pump, $p$, signal, $s$, and idler, $i$. To obtain the field overlap integrals, the modes of the hybrid PCF are calculated with a full-vector modal solver based on the finite element method, 50 modes for each considered wavelength. In Fig. 1d the gain has been calculated for signal and idler being generated in the fundamental mode. Similar gain curves for other combinations are considered, and the combination resulting in the largest gain can be found, which allows one to predict which modes the signal and idler will be generated in, in an actual measurement.

\section{References}

1. J. C. Knight, J. Arriaga, T. A. Birks, A. Ortigosa-Blanch, W. J. Wadsworth, and P. St. J. Russell "Anomalous Dispersion in Photonic Crystal Fiber," IEEE Photon. Technol. Lett. 12,(7) 807-809 (2000).

2. T. T. Alkeskjold, "Large-mode-area ytterbium-doped fiber amplifier with distributed narrow spectral filtering and reduced bend sensitivity," Opt. Express 17,(19) 16394-16405 (2009).

3. J. Hansryd and P. A. Andrekson, "Fiber-Based Optical Parametric Amplifiers and Their Applications," IEEE J. Sel. Topics Quantum Electron. 8,(3) 506-520 (2002).

4. Govind P. Agrawal, "Nonlinear Fiber Optics,” Elsevier, United States of America, Fourth edition, 2007. 